\title{
Search for new neutral gauge bosons with the CMS Experiment at the LHC
}

\author{
Alexander Lanyov ${ }^{1, \star}$, Sergei Shmatov ${ }^{1, \star \star}$, and Ilia Zhizhin ${ }^{1,2, \star \star \star}$ \\ ${ }^{1}$ Joint Institute for Nuclear Research, Joliot-Curie 6, 141980 Dubna, Russia \\ ${ }^{2}$ Dubna State University, Universitetskaya 19, 141982 Dubna, Russia
}

\begin{abstract}
A search for narrow resonances in dimuon invariant mass spectra has been performed using $13 \mathrm{fb}^{-1}$ data obtained in 2016 from proton-proton collisions at $\sqrt{s}=$ $13 \mathrm{TeV}$ with the CMS experiment at the LHC. No evidence for physics beyond standard model is found. Limits on the production cross section and the masses of hypothetical particles that could appear in the scenarios of new physics have been set.
\end{abstract}

\section{Introduction}

The CMS physics research programme on the detection of new physics beyond the standard model $(\mathrm{SM})$ is aimed at detecting deviations from SM predictions in various channels [1]. The CMS searches signals from new physical objects and phenomena predicted by supersymmetric models [2], models of the extended gauge sector [3], models of technicolor [4], scenarios of extra-dimensions theories [5], and to solve the hierarchy problems [6,7] (fine tuning of SM) and many others. The variety of new particles and phenomena predicted by these models requires the use of a wide range of experimental studies in various channels:

- Heavy resonance states and nonresonant signals in the spectrum of pairs of leptons, jets, photons, top quarks, gauge bosons to search for new heavy gauge bosons $Z^{\prime}$ [3], Kaluza-Klein excited states of the graviton [8], technicolor particles [4], etc.

- The single production of particles (jets, photons, leptons), accompanied by a large fraction of the missing transverse energy. A deviation in the behavior of such signals from SM can indicate an existence of a new heavy charged gauge boson $W^{\prime}$, extra spatial dimensions $[9,10]$, weakly interacting particles of dark matter [11].

Many physical scenarios outside the framework of the SM predict the existence of new resonance states in the spectra of the SM particles [12]. For example, heavy (with a mass of several TeV) resonances in the spectrum of pairs of leptons and jets appear in four-dimensional models with an extended gauge sector (extra gauge boson $Z^{\prime}$ beyond the SM with spin $S=1$ ). In its characteristics, $Z^{\prime}$ is similar to the usual SM neutral gauge boson $Z$, the main differences are the larger expected mass and different coupling constants. The extra-dimension gravity scenario Randall-Sundrum (RS1) predicts other massive (also with $\mathrm{m} \sim \mathrm{TeV}$ ) particles — the Kaluza-Klein excitations (KK modes) of

\footnotetext{
^e-mail: alexander.lanyov@cern.ch

$\star \star$ e-mail: sergei.shmatov@cern.ch

$\star \star \star$ e-mail: ilia.zhizhin@cern.ch
} 
the graviton $G_{\mathrm{KK}}$ (RS graviton) [9, 10]. Due to a different spin value ( $\left.S=2\right)$, RS gravitons can decay not only into leptons and jets originated from quarks or gluons, but also into two SM gauge bosons $(\gamma \gamma, \mathrm{WW}, \mathrm{ZZ})$. One of the experimental signatures of these processes can be a pair of leptons, photons or jets with large transverse momenta from the same vertex [1].

In this paper, we will give results of the search for new heavy resonances outside the framework of the SM, using the data obtained by the CMS experiment at $\sqrt{s}=13 \mathrm{TeV}$ on statistics corresponding to the integral luminosity of $13 \mathrm{fb}^{-1}$ in 2016 [13].

\section{CMS detector}

The search for new particles is carried out using the CMS detector (Compact Muon Solenoid), one of the four main LHC detectors [14, 15, 17]. It has an overall length of $22 \mathrm{~m}$, a diameter of $15 \mathrm{~m}$, and weighs 14000 tonnes. The central feature of the CMS apparatus is a superconducting solenoid of $6 \mathrm{~m}$ internal diameter, providing a magnetic field of 3.8 T. Within the solenoid volume are a silicon pixel and strip tracker, a lead tungstate crystal electromagnetic calorimeter, and a brass and scintillator hadron calorimeter, each composed of a barrel and two endcap sections. Muons are detected in gas-ionization chambers embedded in the steel flux-return yoke outside the solenoid, in the pseudorapidity range $|\eta|<2.4$, with detection planes made using three technologies: drift tubes, cathode strip chambers, and resistive plate chambers. Matching muons to tracks measured in the silicon tracker results in a relative transverse momentum resolution for muons with $20<p_{T}<100 \mathrm{GeV}$ of $1.3-2.0 \%$ in the barrel $(|\eta|<1.2)$ and better than $6 \%$ in the endcaps $(0.9<|\eta|<2.4)$, the $p_{T}$ resolution in the barrel is better than $10 \%$ for muons with $p_{T}$ up to $1 \mathrm{TeV}$ [15]. Events of interest are selected using a two-tiered trigger system [16]. The first level (L1), composed of custom hardware processors, uses information from the calorimeters and muon detectors to select events at a rate of around $100 \mathrm{kHz}$ within a time interval of less than $4 \mu \mathrm{s}$. The second level, known as the high-level trigger (HLT), consists of a farm of processors running a version of the full event reconstruction software optimized for fast processing, and reduces the event rate to around $1 \mathrm{kHz}$ before data storage. A more detailed description of the CMS detector can be found in Ref. [14].

\section{Dimuon resonances}

In the CMS analysis the typical models [3] predicting extra Z-like bosons decaying to muon pairs have been considered. These models extend the gauge group of the SM by additional $U^{\prime}(1)$ gauge groups. The $U^{\prime}(1)$ gauge groups, or a linear combination of them, are maybe broken near the TeV scale giving rise to massive $Z^{\prime}$ gauge bosons. These models are generalized using an angle that continuously parametrizes the mixing of the respective $U^{\prime}(1)$ generators. Specific choices of the mixing angle produce different models. One of them, the so-called Sequential Standard Model (SSM) in which heavy bosons $\left(Z_{S S M}^{\prime}\right.$ and $\left.W_{S S M}^{\prime}\right)$ are assumed to have the same coupling constants as for $Z$ and $W$ bosons in the SM. Another one, $\psi$-model, is a special case of an extension of the SM to one of the gauge groups of the grand unification theory [3].

\section{Event selection}

The reconstruction algorithms and event selection employed are refined versions of those used for previous high-mass dilepton searches [18]. To reconstruct a muon candidate, hits from the inner tracker and the muon system are first fitted separately to have inner tracker and standalone muon tracks and then combined in a global muon track hypothesis. 
Candidate muon pair events are required to have at least one muon with track segments reconstructed in the muon detectors and with transverse momentum $p_{T}$ above $16 \mathrm{GeV}$ at L1 trigger, and at least one muon with $p_{T}>50 \mathrm{GeV}$ and $|\eta|<2.4$ at the HLT. In order to extract the normalization factor in the control region $\left(60<m_{l l}<120 \mathrm{GeV}\right)$ around the $Z$ peak, a prescaled HLT trigger with a $p_{T}$ threshold of $27 \mathrm{GeV}$ is used without changing other $\mathrm{L} 1$ requirements. The muon candidates are required to have $p_{T}>53 \mathrm{GeV}$ and be within the sensitive region of $|\eta|<2.4$.

The muon candidates are also required to pass a dedicated high-momentum muon identification selection [19]. Finally, isolated muon candidates are selected by requiring the $p_{T}$ sum of tracks within a cone of radius $\Delta R=\sqrt{(\Delta \eta)^{2}+(\Delta \phi)^{2}}<0.3$ around the candidate direction to be less than $10 \%$ of the $p_{T}$ of the candidate. The sums exclude the lepton candidate under consideration.

The selected opposite-charge muons are combined to form dimuon candidates. To ensure that the two muons originate from the same vertex a fit is performed to a common vertex. This vertex fit is required to have $\chi^{2} /$ dof $<20$. To suppress background from cosmic ray muons that pass near the interaction point, the three-dimensional angle between the two track momentum vectors is required to be less than $\pi-0.02$. In case several dimuon pairs are found in an event, only the one with the two highest- $p_{T}$ muons is retained.

Description of the dielectron selection can be found in $[13,20]$.

\section{Analysis strategy}

The search for new resonances was carried out by a combined analysis of the shape of muon pair invaraiant mass distributions. The analysis in the muon channel is performed in three different categories to isolate detector alignment and calibration effects in the endcap region, while have a category with the better resolution and calibration that provides higher sensitivity to new physics. Three categories are defined on this purpose considering events with: two muons in the barrel region $(|\eta|<1.2)$; at least one muon in the negative endcap $(\eta<-1.2)$; one muon in the barrel region and one muon in the positive endcap $(\eta>1.2)$. This separation allows the assignment of separate uncertainties to each category.

Using a Bayesian approach with an unbinned extended likelihood function, limits are derived for the production of a narrow spin-1 resonance $[13,21]$. The likelihood function is based on probability density functions (pdf) describing the invariant mass spectra as a sum of signal and background pdfs.

The signal pdf is modeled as the convolution of a non relativistic Breit-Wigner (BW) with a Gaussian that accounts for the detector resolution effects. This analysis is designed for narrow resonances having a small BW intrinsic width $(\Gamma)$ compared to the detector resolution. For such narrow widths, variations in $\Gamma$ have typically little effect on the derived limits. The intrinsic widths of the $Z_{S S M}^{\prime}$ and $Z_{\psi}^{\prime}$ resonances are $3 \%$ and $0.6 \%$, respectively. The resolutions are predicted by Monte Carlo (MC) as a function of the generated dilepton mass and are adjusted using $Z$ boson and cosmic ray data events. The resolution for dimuon (dielectron) pairs with a mass of $2 \mathrm{TeV}$ is $5.5 \%$ (1\%) for barrel-barrel pairs, $8.5 \%(1.5 \%)$ for barrel-endcap pairs. [13, 21]

The functional form of the background pdf is defined by considering the complete background. The dominant and irreducible SM background to a $Z^{\prime}$ decaying to a muon or electron pair arises from Drell-Yan $Z / \gamma^{*} \rightarrow l^{+} l^{-}$process. The irreducible $Z / \gamma^{*} \rightarrow l^{+} l^{-}$background and real prompt leptons backgrounds such as $t \bar{t}$, tW, pair production of gauge bosons (ZZ, ZW, WW) are estimated using MC simulated events. 


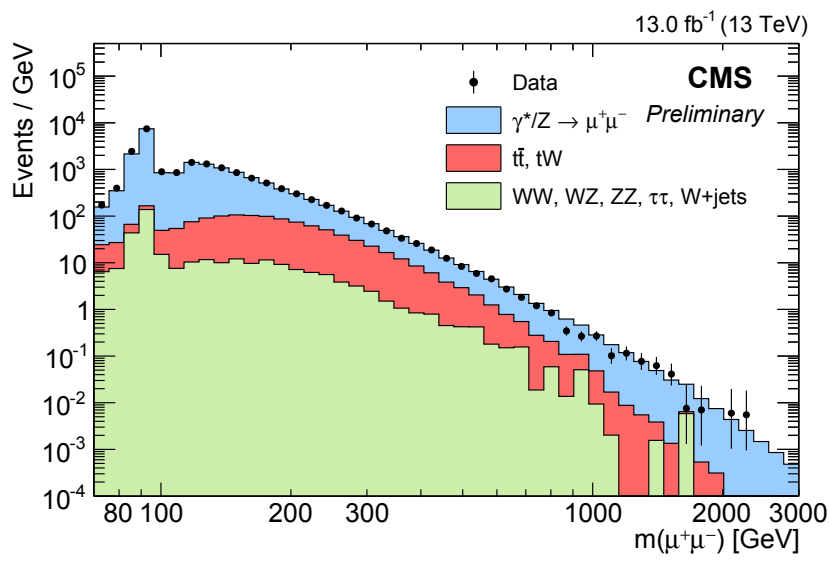

Figure 1. Invariant mass spectrum of dimuon events at $\sqrt{s}=13 \mathrm{TeV}$.

\section{Analysis and results}

Search for narrow heavy dimuon resonances has been performed with the data recorded in 2016, corresponding to an integrated luminosity of $13 \mathrm{fb}^{-1}$ at $\sqrt{s}=13 \mathrm{TeV}$ [13]. The dimuon invariant mass spectrum is shown in Fig. 1. To calculate the mass limits, normalization to $\sigma(Z)$ is performed:

$$
R_{\sigma}=\frac{\sigma\left(Z^{\prime} \rightarrow \mu^{+} \mu^{-}\right)}{\sigma\left(Z \rightarrow \mu^{+} \mu^{-}\right)}=\frac{N\left(Z^{\prime}\right)}{N(Z)} \times \frac{A(Z)}{A\left(Z^{\prime}\right)} \times \frac{\varepsilon(Z)}{\varepsilon\left(Z^{\prime}\right)}
$$

In this way the luminosity uncertainty is removed and other systematic effects reduced. The search for resonances is based on a shape analysis of dilepton mass spectra, in order to be robust against uncertainties in the absolute background level. Existence of signal is established by performing unbinned maximum likelihood fits to the observed spectrum. No significant deviations from the SM expectation have been observed, therefore lower bounds are set on the masses of hypothetical particles that arise in new-physics scenarios. The procedure of setting the confidence level by maximization of the Bayes likelihood function using the Metropolis-Hastings method [22] was similar to the analysis at $8 \mathrm{TeV}$ [19]. Using the dimuon analysis, we could exclude at $95 \%$ C.L. the following $Z^{\prime}$ masses: the $Z_{\mathrm{SSM}}$ can be excluded below $3.75 \mathrm{TeV}$, the $Z_{\psi}$ below $3.20 \mathrm{TeV}$. Combining the measurements from both dimuon and electron channels (Fig. 2), the limits for production cross section at $\sqrt{s}=13 \mathrm{TeV}$ for $Z^{\prime}$ were improved: they exclude a $Z_{S S M}^{\prime}$ with a mass less than $4.0 \mathrm{TeV}$ and $Z_{\psi}^{\prime}$ with a mass less than $3.5 \mathrm{TeV}$ [13].

\section{Conclusions}

The CMS Collaboration has searched for narrow resonances in the invariant mass spectrum of dimuon and dielectron final states in the event samples corresponding to an integrated luminosity of $13 \mathrm{fb}^{-1}$ at $\sqrt{s}=13 \mathrm{TeV}$. This search channel benefits from high signal selection efficiencies and relatively small, well-understood backgrounds. The spectra are consistent with expectations from the SM and the upper limits have been set on the cross section times branching fraction for $Z^{\prime}$ into lepton pairs relative to $\mathrm{SM} Z$ boson production.

We would like to thank the management of the CMS theme at JINR for support. 


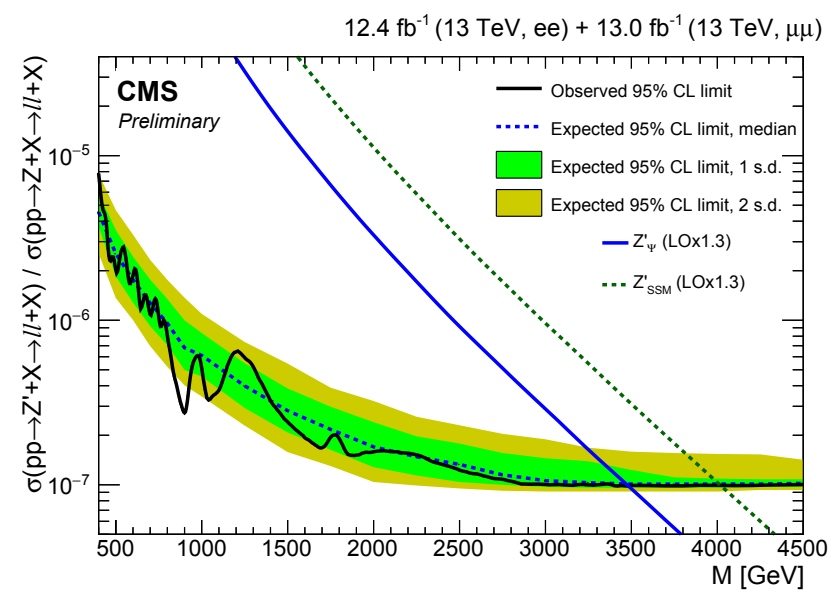

Figure 2. The cross section limits as a function of dilepton mass. Black curve is data, bands around the curve show the expected limits. The solid curve shows the theoretical prediction of cross section for $Z_{\psi}^{\prime}$ model as a function of dilepton mass and dotted curve corresponds to the prediction for $Z_{S S M}^{\prime}$ model.

\section{References}

[1] CMS Collaboration, CMS Physics TDR, Vol. II: Physics Performance, J. Phys. G 34, 995 (2007).

[2] H. Baer, M. Brhlik, C. H. Chen, and X. Tata, Phys. Rev. D 55, 4463 (1997).

[3] A. Leike, Phys. Rept. 317, 143 (1999).

[4] L. Susskind, Phys. Rev. D20, 2619 (1979).

[5] V. A. Rubakov, Phys. Usp. 44, 871 (2001).

[6] N. Arkani-Hamed, S. Dimopoulos, and G. Dvali, Phys. Lett. B 429, 263 (1998).

[7] N. Arkani-Hamed, S. Dimopoulos, and G. Dvali, Phys. Rev. D 59, 086004 (1999).

[8] J. Hewett, Phys. Rev. Lett. 82, 4765 (1999).

[9] L. Randall and R. Sundrum, Phys. Rev. Lett. 83, 4690 (1999).

[10] L. Randall and R. Sundrum, Phys. Rev. Lett. 83, 3370 (1999).

[11] V. A. Ryabov, V. A. Tsarev, and A. M. Tskhovrebov, Phys. Usp. 51, 1091 (2008)

[12] A. V. Lanyov, Phys. Usp. 57, 923 (2014).

[13] CMS Collaboration, CMS-PAS-EXO-16-031 (2016).

[14] CMS Collaboration, JINST 3, S08004 (2008).

[15] CMS Collaboration, JINST 8, P11002 (2013).

[16] CMS Collaboration, JINST 12, P01020 (2017).

[17] CMS Collaboration, CMS Physics TDR, Vol. I: Detector Performance and Software, CERN/LHCC 2006-001 (2006).

[18] CMS Collaboration, Phys. Lett. B768, 57 (2017).

[19] CMS Collaboration, JHEP 04, 025 (2015).

[20] CMS Collaboration, JINST 10, P06005 (2015).

[21] E. Accomando, A. Belyaev, L. Fedeli et al., Phys. Rev. D83, 075012 (2011).

[22] W. Hastings, Biometrika 57, 97 (1970). 